\title{
Análise de discrepância nas prescrições dos pacientes admitidos no Centro Cirúrgico de um Hospital Público de Urgência
}

Discrepancy analysis in the prescriptions of patients admitted to the Surgical Center of a Public Emergency Hospital

Análisis de discrepancias en la prescripción de pacientes ingresados en el Centro Quirúrgico de un Hospital Público de Urgencias

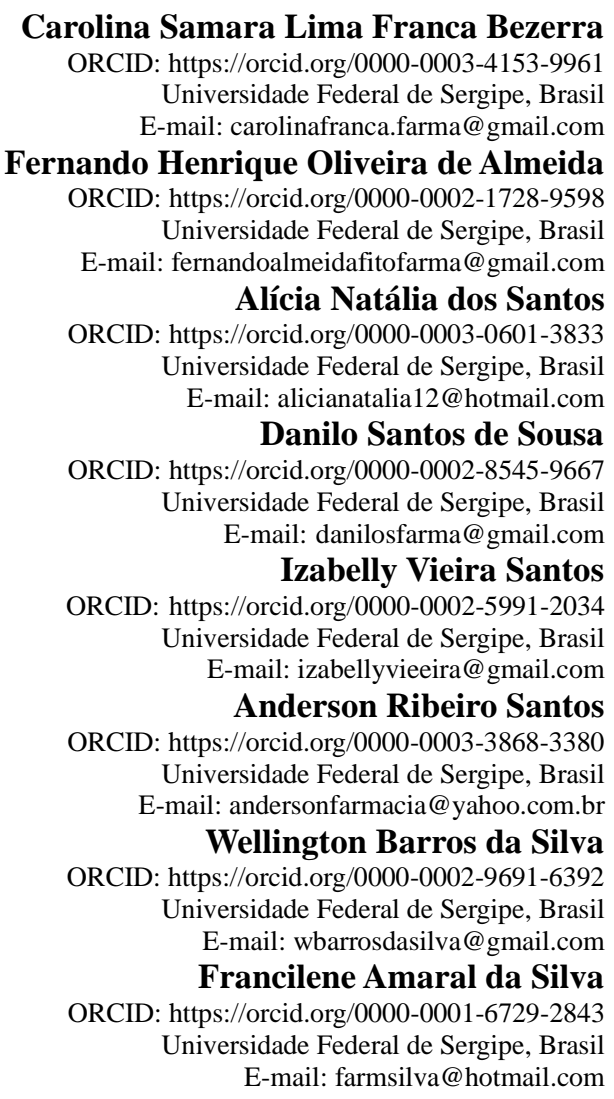

\section{Resumo}

A reconciliação medicamentosa tem forte impacto na prevenção de eventos adversos e equívocos relacionados aos medicamentos. O objetivo do estudo foi observar a ocorrência de divergências na farmacoterapia de pacientes cirúrgicos. Considerou-se pacientes polimedicados, aqueles que utilizavam três ou mais medicamentos. As fontes utilizadas foram o próprio paciente, seu acompanhante, os medicamentos em uso domiciliar levados ao hospital e a consulta ao prontuário. As discrepâncias eram consideradas como qualquer mudança não intencional entre os fármacos usados previamente a admissão e os medicamentos prescritos após a intervenção cirúrgica, podendo ser: omissão de medicamentos, diferenciação nas doses, divergências na frequência/horário de administração, interação medicamentosa e duplicidade terapêutica. Os dados coletados foram tabulados e estudados. Durante o trabalho, 422 pacientes deram entrada no internamento pós-cirúrgico. O maior motivo de internação foi amputação de membro inferior ou superior (41,3\%); a média de fármacos usadas no domicílio foi 4,7. Um total de 444 discrepâncias foi levantado e a média por paciente foi de 5,5. As mais relevantes foram interação, equivalendo a 53,4\% $(\mathrm{n}=237)$ de todas as discrepâncias identificadas, depois foi a omissão sendo 25,7\% $(n=114)$. Das 106 intervenções somente 15 foram aceitas. As discrepâncias encontradas indicam alto risco para segurança desses pacientes, sendo preciso um processo eficaz de reconhecimento da terapia domiciliar quando o indivíduo é admitido na internação. Dessa forma, medidas que garantam a segurança do paciente na admissão hospitalar e atenuem os equívocos de medicamentos devem ser praticadas.

Palavras-chave: Centro cirúrgico hospitalar; Polimedicação; Reconciliação de medicamentos; Segurança do paciente. 


\begin{abstract}
Medication reconciliation has a strong impact on the prevention of adverse events and mistakes related to medications. The study aimed to observe the occurrence of divergences in the pharmacotherapy of surgical patients. Polymedicated patients were considered as those who used three or more medications. The sources used were the patient himself, his companion, the drugs taken at home taken to the hospital, and the consultation of the medical record. Discrepancies were considered as any unintentional change between the drugs used before admission and the drugs prescribed after the surgical intervention, which could be: omission of drugs, differentiation in doses, differences in frequency/time of administration, drug interaction, and therapeutic duplication. The collected data were tabulated and studied. During the work, 422 patients were admitted to post-surgical hospitalization. The main reason for admission was lower or upper limb amputation (41.3\%); the average number of drugs used at home was 4.7 . A total of 444 discrepancies were found and the average per patient was 5.5. The most relevant were interactions, equivalent to $53.4 \% \quad(n=237)$ of all identified discrepancies, then omission was $25.7 \% \quad(n=114)$. Of the 106 interventions, only 15 were accepted. The discrepancies were found to indicate a high risk for the safety of these patients, requiring an effective process of recognition of home therapy when the individual is admitted to the hospital. Thus, measures to ensure patient safety at hospital admission and mitigate drug errors should be taken.
\end{abstract}

Keywords: Medication reconciliation; Patient safety; Polypharmacy; Surgery department, hospital.

\title{
Resumen
}

La conciliación de medicamentos tiene un fuerte impacto en la prevención de eventos adversos y errores relacionados con los medicamentos. El objetivo del estudio fue observar la ocurrencia de divergencias en la farmacoterapia de pacientes quirúrgicos. Se consideraron pacientes polimedicados aquellos que utilizaron tres o más medicamentos. Las fuentes utilizadas fueron el propio paciente, su acompañante, los medicamentos que tomaba en su domicilio y los llevaban al hospital y la consulta de la historia clínica. Se consideró discrepancias cualquier cambio no intencionado entre los fármacos utilizados antes del ingreso y los fármacos prescritos tras la intervención quirúrgica, que podrían ser: omisión de fármacos, diferenciación de dosis, diferencias en frecuencia / tiempo de administración, interacción farmacológica y duplicación terapéutica. Los datos recopilados se tabularon y estudiaron. Durante el trabajo, 422 pacientes ingresaron para hospitalización posquirúrgica. El principal motivo de ingreso fue la amputación de miembros inferiores o superiores (41,3\%); el número medio de drogas consumidas en casa fue de 4,7. Se encontraron un total de 444 discrepancias y la media por paciente fue de 5,5. Las más relevantes fueron la interacción, equivalente al $53,4 \%(n=237)$ de todas las discrepancias identificadas, luego la omisión fue del 25,7\% ( $\mathrm{n}=114)$. De las 106 intervenciones, solo se aceptaron 15. Las discrepancias encontradas indican un alto riesgo para la seguridad de estos pacientes, requiriendo un proceso efectivo de reconocimiento de la terapia domiciliaria cuando el individuo ingresa en el hospital. Por lo tanto, se deben tomar medidas para garantizar la seguridad del paciente al ingreso hospitalario y mitigar los errores de medicación.

Palabras clave: Conciliación de medicamentos; Polifarmacia; Seguridad del paciente; Servicio de cirugía en hospital.

\section{Introdução}

As divergências medicamentosas são responsáveis por fortes danos aos pacientes, sobretudo em âmbito hospitalar.

Esses erros normalmente acontecem por equívocos de prescrições se relacionando aos históricos de medicação incompletos. Estas lacunas que resultam em erros vão desde a admissão do paciente no hospital até a sua saída. É notável, que, facilmente, tal atitude leva à divergência entre os medicamentos usados durante esse intervalo de tempo, podendo acarretar danos à saúde do indivíduo assistido. Sabe-se que, 50\% das discrepâncias ocorrem quando os pacientes chegam, recebem alta, são transferidos entre unidades ou transitam entre os níveis de atenção. (Lombardi et al, 2016).

Por isso, faz-se necessário que, no momento da admissão, exista um diálogo preciso entre o profissional de saúde e o paciente e/ou familiares a fim de averiguar e listar os medicamentos de uso daquele paciente e comparar com a prescrição que está sendo feita no hospital, seja na admissão, na mudança de setor ou na alta. Nessa análise deve conter o nome, a dosagem, a frequência e a via de administração das substâncias de uso. Além disso, em caso de mudança de profissional de outro setor ou nível de cuidado, deve-se informar toda a lista de medicamentos. E, por fim, antes da alta do paciente, orientar e assegurar o entendimento a respeito de todo o tratamento que o mesmo fará em casa. A esse processo, dá-se o nome de reconciliação medicamentosa. (Mendes et al, 2016; Salanitro et al, 2013).

Assim, a reconciliação medicamentosa é apontada por diversos estudos como uma alternativa para evitar o desconforto e proporcionar a segurança do paciente, que, segundo a Organização Mundial da Saúde (OMS), corresponde à 
redução ao mínimo aceitável do risco de dano desnecessário associado ao cuidado da saúde. Além da redução de danos à saúde, esse processo é capaz de diminuir o custo financeiro do hospital, pois acaba por evitar gastos desnecessários como a piora do quadro dos pacientes. (Oliveira et al, 2018; Pimenta et al, 2020).

Desse modo, faz-se necessário analisar as discrepâncias encontradas em pacientes submetidos a procedimento cirúrgico num hospital de urgência e emergência do estado de Sergipe e realizar a tentativa de reconciliação medicamentosa dos casos discrepantes.

\section{Metodologia}

Este estudo é observacional do tipo exploratório descritivo, de delineamento transversal, no HUSE (Hospital de Urgências de Sergipe). Hospital este, completamente integrado ao SUS (Sistema Único de Saúde) que assiste urgência e emergência.

Considerou-se como critério de inclusão: pacientes admitidos no Internamento Cirúrgico, que ficassem mais de 24 horas internados, que consentisse participar do estudo e usassem três ou mais medicamentos no domicílio. Foram excluídos os pacientes que não foi possível conduzir a entrevista (como era o caso de pacientes que estavam em isolamento, inconscientes ou desacompanhados) e aqueles que não foi possível acessar o prontuário no momento da avaliação.

Foram avaliadas todas as fontes possíveis e disponíveis sobre os medicamentos do paciente no momento da entrevista, tais como: o prontuário, a entrevista com o acompanhante/cuidador, o próprio paciente e seus medicamentos quando o mesmo os levava para o hospital além de prescrições anteriores.

Para que fosse considerado discrepâncias precisava ser uma mudança não justificada entre a história prévia da farmacoterapia do paciente e a prescrição após a admissão no hospital. E desconsiderava-se como discrepância caso houvesse justificativa médica para o motivo da modificação. Os medicamentos classificados como: "prescritos se necessário" também foram avaliados caso apresentasse alguma diferença.

As discrepâncias eram consideradas como qualquer mudança não intencional entre os fármacos usados previamente a admissão e os medicamentos prescritos após a intervenção cirúrgica, podendo ser: omissão de medicamentos, diferenciação nas doses, divergências na frequência/horário de administração, interação medicamentosa e duplicidade terapêutica. Foi registrado ainda as origens das discrepâncias encontradas, sendo elas de dados do prontuário, do relato do paciente, de exposição do acompanhante/cuidador ou da averiguação dos medicamentos levados ao hospital.

Após elencar as discrepâncias, as intervenções requeridas foram registradas em prontuário, resultando em um documento para notificar o profissional prescritor contendo as informações adquiridas, afim de resultar em uma intervenção e acompanhamento, bem como observada a aceitação do médico prescritor. A intervenção foi considerada "aceita" se a proposta do farmacêutico pesquisador foi acatada pelo médico e, "não aceita" se não houve alteração da prescrição após apresentação da proposta.

Os dados levantados foram tabulados em planilha do Excel e demonstrados por meio de proporções, média, desvio padrão e intervalo de confiança. $O$ teste de qui-quadrado foi realizado para as associações entre os dados e foi considerado um nível de significância de 0,05. Para isso, o programa estatístico SPSS (Statistical Package for Social Sciences) foi escolhido para o trabalho. A Diretoria do Hospital e Comitê de Ética em Pesquisa da Universidade Federal de Sergipe, autorizou o estudo por meio do registro CAAE na Plataforma Brasil n ${ }^{\circ} 34038114.7 .0000 .5546$.

\section{Resultados}

Durante o tempo do estudo, 422 pacientes deram entrada nas quatro unidades de internação cirúrgica do hospital. Somente 80 dos 422 atenderam aos critérios de inclusão no momento da admissão. Ficaram excluídos 07 pacientes, por 
motivos de: $n=04(0,95 \%)$ pacientes estavam inconscientes ou incapazes de dar o seu consentimento à pesquisa, $n=03(0,71 \%)$ pacientes que não sabiam ou não tinham fonte adicional da história dos seus medicamentos em suas casas.

A média de idade dos 422 pacientes era de 44,05 anos \pm 21,0 (12-98). Destes, 295 (69,9\%) eram homens e 127 $(30,1 \%)$ mulheres. Houve associação estatisticamente significativa entre a idade dos pacientes e o número de discrepâncias $\left(X^{2}=4,76 ; p=0,02\right)$. Contudo, o sexo e o número de discrepâncias $\left(X^{2}=1,37 ; p=0,24\right)$ não houve associação estatisticamente significativa. Dentre os motivos de internação dos pacientes, os mais relevantes foram: fratura de membro inferior ou superior, $173(41,0 \%)$, seguido de cirurgia no abdômen 79 (18,7\%) e ablação de membros inferiores ou superiores 57 (13,5\%). A Tabela 1 representa as características sóciodemográficas dos pacientes admitidos no hospital.

Tabela 1. Características sociodemográficas dos pacientes (N=80) - HUSE/SE. Brasil.

\begin{tabular}{ll}
\hline Características & \\
\hline SEXO & $43(53,8 \%)$ \\
Feminino (\%) & $37(46,3 \%)$ \\
Masculino (\%) & 64,4 anos $( \pm 17,0)$ \\
IDADE MÉDIA (DP) & $(23-93)$ \\
GESTÃO DOS MEDICAMENTOS & $58(72,5 \%)$ \\
Por familiares (\%) & $22(27,5 \%)$ \\
Pelo próprio paciente (\%)
\end{tabular}

Fonte: Autoria própria.

Foi entrevistando a maioria dos próprios pacientes que a as informações referentes aos dados clínicos e levantamento do histórico medicamentoso foram elencadas $(n=75 ; 93,8 \%)$, quando não foi possível, entrevistou-se o acompanhante ou cuidador responsável $(n=64 ; 80,0 \%)$, além disso, houve consulta através dos prontuários $(n=13 ; 16,3 \%)$. Por fim, foi possível observar que $38(47,5 \%)$ pacientes levaram os medicamentos que usavam antes da internação para o hospital.

Dos $80(19 \%)$ pacientes, $43(53,8 \%)$ eram do sexo feminino e $37(46,3 \%)$ do sexo feminino, possuindo média de idade igual a 64,14 anos $\pm 17,0$ (23-93). No que tange à gestão dos medicamentos, em 58 (72,5\%) casos ficava a cargo dos familiares e em $22(27,5 \%)$ era feita pelo próprio paciente. Foram prescritos um total de 830 medicamentos, sendo dipirona $(n=75 ; 9,0 \%)$ o medicamento mais prevalente, seguido de tramadol $(n=71 ; 8,5 \%)$ e enoxaparina $(n=65 ; 7,8 \%)$.

Por conta do grande de número de pacientes acometidos por doenças crônicas como a diabetes, um dos motivos mais prevalentes de internação desses pacientes era a amputação do membro inferior ou superior 33 (41,3\%), podendo ser o motivo decorrente de algum trauma nessa região também.

A média de medicamentos usados em casa foi de 4,7 \pm 1,9 (3-14), e foi constatado que grande parte dos pacientes fazia uso de pelo menos três medicamentos. Foi observada associação estatisticamente relevante entre o número de medicamentos utilizados em casa e o número de divergências identificadas $\left(\mathrm{X}^{2}=6,16 ; \mathrm{p}=0,013\right)$. Um montante de 367 medicamentos foi avaliado no domicílio do paciente, entre os mais usados estavam os anti-hipertensivos ( $\mathrm{n}=83 ; 22,6 \%)$, em seguida dos hipoglicemiantes $(n=74 ; 20,2 \%)$ por fim os medicamentos do sangue $(n=37 ; 10,1 \%)$. O quadro 1 mostra outros detalhes das classes terapêuticas de uso domiciliar e hospitalar envolvidas nas discrepâncias. 
Quadro 1. Detalhamento das classes terapêuticas de uso domiciliar e hospitalar envolvidas nas discrepâncias HUSE/SE.

\begin{tabular}{|l|l|l|}
\hline ATC* Nível 1 & $\begin{array}{l}\mathbf{N}^{\mathbf{0}} \text { medicamentos domiciliar } \\
\text { envolvidos em discrepâncias } \\
(\mathbf{n = 3 6 7 )}\end{array}$ & $\begin{array}{l}\mathbf{N}^{\mathbf{0}} \text { medicamentos hospitalar } \\
\text { envolvidos em discrepâncias } \\
(\mathbf{n = 8 3 0})\end{array}$ \\
\hline C - aparelho cardiovascular & $\mathbf{1 2 7}(\mathbf{3 4 , 6 \%})$ & $160(19,3 \%)$ \\
\hline A - aparelho digestivo e metabolismo & $107(29,1 \%)$ & $\mathbf{2 4 3}(\mathbf{2 9 , 3 \%})$ \\
\hline $\mathrm{N}$ - Sistema nervoso & $54(14,7 \%)$ & $189(22,8 \%)$ \\
\hline B - sangue e órgãos hematopoiéticos & $37(10,1 \%)$ & $95(11,4 \%)$ \\
\hline M - sistema músculo-esquelético & $18(4,9 \%)$ & $8(1,0 \%)$ \\
\hline J - anti-infecciosos gerais para uso sistêmico & $12(3,3 \%)$ & $128(15,4 \%)$ \\
\hline Outros (R, S, H) & $12(3,3 \%)$ & $7(0,8 \%)$ \\
\hline
\end{tabular}

*ATC: Sistema de Classificação Terapêutica Anatômica. Fonte: Autoria própria.

Dos pacientes avaliados alguns possuíam várias comorbidades. Foi encontrado um total de 153, dentre elas diabetes $(n=40 ; 18,6 \%)$, doenças no aparelho cardiovascular $(n=31 ; 14,4 \%)$, sendo uma delas a hipertensão $(n=28 ; 13,0 \%)$. Entre a quantidade de comorbidades e os medicamentos usados em casa não houve associação estatisticamente significante $\left(\mathrm{X}^{2}=1,41\right.$; $\mathrm{p}=0,23)$.

Durante a admissão hospitalar os pacientes submetidos a reconciliação $(n=80)$ apresentaram uma ou mais discrepâncias na farmacoterapia, no total foram identificadas 144 divergências, com média de 5,5 $\pm 2,78$ (1-14) por paciente.

As discrepâncias mais relevantes foram interação, que significou mais de $50 \%$ de todas as discrepâncias identificadas, sendo 53,4\% $(\mathrm{n}=237)$ e a omissão que ficou responsável por 25,7\% $(\mathrm{n}=114)$ de todas as discrepâncias. Em seguida estavam as discrepâncias por duplicidade terapêutica $(12,6 \%$; $=56)$, de horário ou frequência $(5,6 \%$; $n=25)$ e de dose $(2,7 \% ; \mathrm{n}=12)$, expressas na Figura 1.

Figura 1. Discrepâncias identificadas no estudo- HUSE/SE. Brasil: agosto - fevereiro (2014-2015).

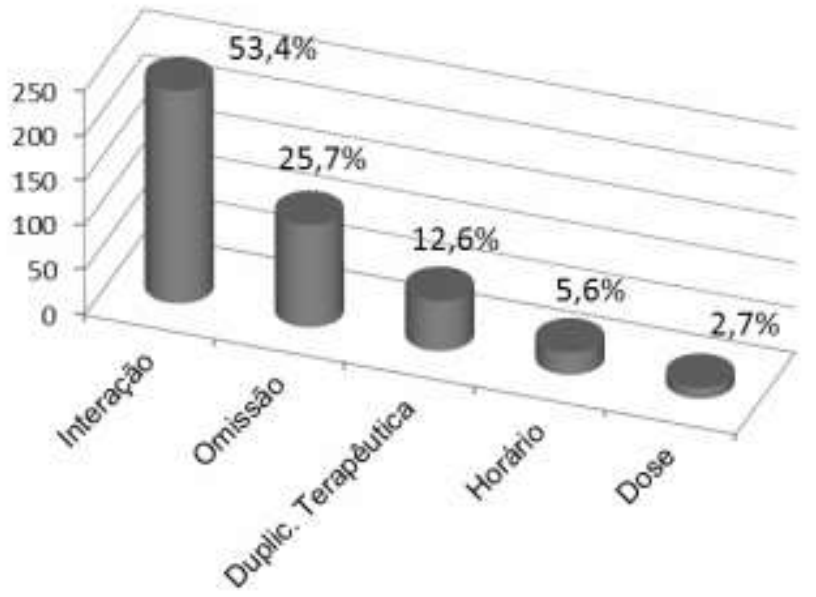

Fonte: Autoria própria.

A Tabela 2 mostra o número de pacientes envolvidos nas discrepâncias encontradas. 
Tabela 2. Distribuição de pacientes por tipo de discrepância - HUSE/SE.

\begin{tabular}{ll}
\hline Discrepâncias & Pacientes envolvidos (\%) \\
\hline Interação & $72(90,0 \%)$ \\
Duplicidade Terapêutica & $56(70,0 \%)$ \\
Omissão & $54(67,5 \%)$ \\
Frequência/ Horário & $20(25,0 \%)$ \\
Dose & $9(11,3 \%)$ \\
\hline
\end{tabular}

Fonte: Autoria própria.

Foram encontradas 237 interações entre medicamentos não poderiam ser utilizados simultaneamente (ex.: tramadol + metoclopramida - pois aumenta o risco de convulsões e pode causar reações extrapiramidais, ou ainda a associação do Ciprofloxacino + insulina - Risco de alterações glicêmicas) e 215 (91\%) eram importantes, ou seja, a interação podia ser perigosa à vida e/ou precisaria de intervenção para atenuar ou evitar efeitos adversos graves (ex.: amitriptilina + metoclopramida e haloperidol + metoclopramida - pois eleva o risco de reações extrapiramidais ou síndrome neuroléptica maligna).

Após elencar e avaliar as discrepâncias, um documento padrão foi elaborado e enviado ao prescritor e à equipe de cuidados, demonstrando as discrepâncias encontradas e os possíveis manejos terapêuticos. Essas informações foram fixadas ao prontuário do paciente e monitorado pelos estudantes da pesquisa se teria ou não a "ciência" do prescritor através da sua assinatura (para ser considerado aceito). Um total de 106 intervenções foram sugeridas, contudo, nenhuma foi assinada pelo prescritor como "ciente", porém algumas modificações nas prescrições foram notadas após a intervenção farmacêutica, dessa forma, foi tida como intervenção aceita $(n=15 ; 14,2 \%)$. Os dados estão demonstrados na Figura 2.

Figura 2. Intervenções Farmacêuticas recomendadas e aceitas pelo prescritor - HUSE/SE.

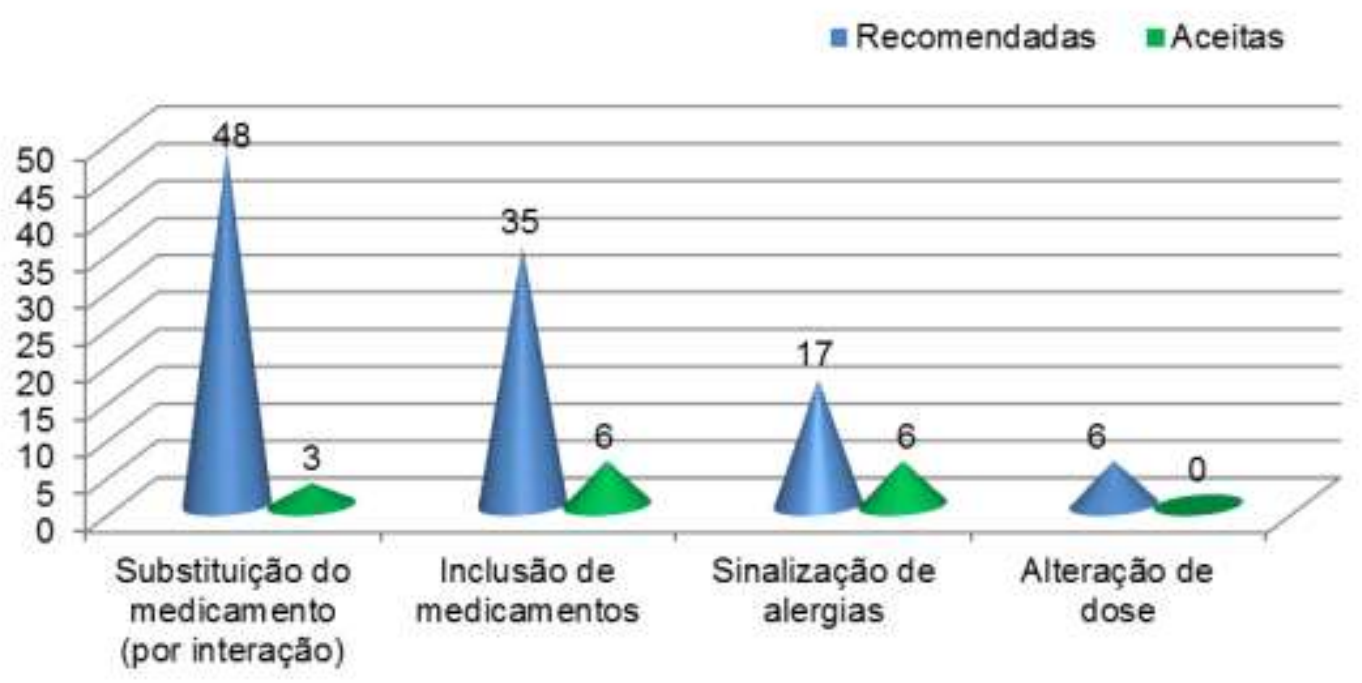

Fonte: Autoria própria.

\section{Discussão}

A característica dos motivos de internamento exposta nos resultados nos aponta a problemática descrita há muito tempo no documento de referência para o Programa Nacional de Segurança do Paciente, elaborado pelo ministério da saúde em 
2014, de que os pacientes admitidos em hospital para um procedimento em particular, como é o caso de uma cirurgia, estão sob cuidados de médicos especialistas em uma área, o que os faz focados em um único componente de cuidado, relacionados a uma prática específica. Dessa forma, a visão do mesmo fica limitada e por consequência não se abre às outras demandas do paciente. Sendo assim, alguns medicamentos podem não ser considerados e por isso causarem algum efeito adverso quando combinados com novos medicamentos prescritos no ambiente hospitalar.

Corroborando com essa ideia e ajudando a resolver o problema, Flamme-Obry (2018) aponta, baseado em outros estudos, a importância do farmacêutico no manejo de pacientes hospitalizados, sobretudo na revisão da farmacoterapia. Além disso, já é evidente que, quando farmacêuticos ou técnicos de farmácia estão envolvidos nesse processo, ocorrem menos erros de medicação. (Markovic et al., 2017).

Este trabalho revelou que a presença de discrepâncias é prevalente no hospital estudado. De acordo com a literatura, as discrepâncias estão relacionadas a erros de medicação e estas podem trazer riscos aos pacientes (Drenth-van Maanen et al, 2011; Quélennec et al., 2013).

Este trabalho revelou que a presença de discrepâncias é prevalente no hospital estudado. Os pacientes admitidos no internamento pós-cirúrgico têm como motivo principal a fratura de membros inferiores ou superiores (41\%). No entanto, diferente dessa prevalência, os pacientes que participaram do estudo demonstraram em 41,3\% dos casos, que seus motivos de internação resultaram em amputação de membros inferiores ou superiores. Associado a esses dados, as comorbidades dos pacientes entrevistados demonstraram que a maior parte deles possuem diabetes e doenças cardiovasculares, incluindo a hipertensão.

Esse dado nos sugere uma relação quando comparado às classes terapêuticas mais utilizadas no domicílio, em que se destacaram os anti-hipertensivos ( $\mathrm{n}=83 ; 22,6 \%)$, seguido dos hipoglicemiantes $(\mathrm{n}=74 ; 20,2 \%)$, acrescidos da média de idade encontrada de 64 anos.

A amputação de membro inferior é duas vezes mais comum em pessoas diabéticas do que em pessoas sem a doença, o que é cerca de $70 \%$ das amputações não traumáticas de membros inferiores, e dentre essas, $85 \%$ destas amputações ocorrem após o surgimento de úlceras, as quais atingem 25\% dos diabéticos. Essa doença atinge 30\% das pessoas acima de 40 anos de idade e as despesas para seu tratamento aumentam significativamente quando o paciente diabético possui amputação de membro (Santos et al, 2018; Peixoto et al, 2017).

Além disso, de acordo com a diretriz da Sociedade Brasileira de Diabetes (2017-2018), a hipertensão arterial é um importante fator no desenvolvimento e progressão das complicações crônicas do diabetes mellitus, e a diminuição das cifras pressóricas tem importante impacto na diminuição dos agravamentos micro e macrovasculares do diabetes mellitus 2, por isso, a hipertensão arterial se comporta como um fator de risco, quando comparada à população em geral sem diabetes.

Quanto às discrepâncias, nossos resultados são consistentes com estudos anteriores que relataram que as discrepâncias de medicamentos na admissão hospitalar são frequentes e são clinicamente significativas (Lombardi et al., 2016; Patel et al., 2018; Pimenta et al., 2020). Um desses estudos revelou que 54,0 \% dos pacientes tinham, pelo menos, um medicamento envolvido na discrepância no momento de internação, que é comparável ao nosso estudo. Na pesquisa de Mendes e seus colaboradores (2016) feita no momento da admissão do paciente de forma randomizada os pacientes reconciliados tiveram uma média de 6 discrepâncias em seus históricos.

Quanto aos fármacos envolvidos nas discrepâncias, os medicamentos que atuam no aparelho cardiovascular constituíram a classe de fármacos mais frequentemente envolvida nas discrepâncias (25,9\%; n=23), o que também foi mostrado no estudo de Unroe e seus colaboradores (2010) no qual os medicamentos cardiovasculares foram os mais encontrados, representando $31 \%$ de todas as discrepâncias na admissão. Outro estudo feito em unidades de cardiologia, mostra que, as maiores discrepâncias acontecem no sistema cardiovascular, sistema nervoso, trato alimentar e metabolismo, e sangue e 
órgãos formadores de sangue, sendo o sistema cardiovascular o mais prevalente. (Lombardi et al., 2016). Entre os fármacos que têm ação no aparelho cardiovascular situam-se os anti-hipertensivos, que em nosso estudo representaram $24,7 \%$ dos medicamentos do domicílio e antilipidêmicos, seguidos dos medicamentos do aparelho digestivo e metabolismo, 24,6\%, que também incluem os hipoglicemiantes orais e insulinas, esses constam uns dos fármacos considerados de alto risco pelo ISMP (Institute for Safe Medication Practices) e NTI (North Carolina Narrow Therapeutic Index).

A discrepância mais prevalente foi a interação entre medicamentos, que representou mais da metade $(53,4 \%)$ da totalidade de discrepâncias identificadas. A omissão constituiu a segunda maior causa das discrepâncias (25,7\%). A falta de questionamentos dos médicos e da equipe de saúde sobre a farmacoterapia anterior dos pacientes pode ser sido uma das principais causas da omissão de medicamentos neste estudo. As omissões causadas pela obtenção incompleta ou imprecisa da história de uso da farmacoterapia podem interromper tratamentos ou levar a indicação de medicamentos inapropriados durante a hospitalização, colocando o paciente em risco (Mueller et al., 2012). Ratificando a necessidade de maior cuidado e acurácia do histórico terapêutico e averiguação da maior quantidade de fontes possíveis a fim de tentar driblar a omissão, um outro estudo mostrou que das 117 discrepâncias encontradas, 50,4\% foram não intencionais e dessas, $61,0 \%$ foram de omissão (Lombardi et al., 2016).

Deficiências e falhas na transição de cuidados, ou seja, nas ações destinadas a assegurar a continuidade dos cuidados de saúde nas transições do paciente, como é o caso da falta de comunicação entre o paciente/familiares e os profissionais de saúde ou entre profissionais de saúde, podem ter contribuído para as discrepâncias encontradas neste estudo. Além disso, estudos mostram que essas omissões ocorrem tanto pelo não questionamento da história da farmacoterapia durante a admissão quanto pela não transcrição no prontuário das informações obtidas durante a entrevista (Allende Bandrés et al., 2013; Mekonnen et al, 2016).

Das intervenções recomendadas (n=106) após identificação e análise das discrepâncias, apenas 15 (14,2\%) foram aceitas. Esse fato aponta para uma das grandes limitações do estudo que é a falta de protocolos organizacionais que estabeleçam processos padronizados no hospital em questão, não somente do serviço de Farmácia, mas também de médicos, enfermeiros, técnicos e auxiliares de enfermagem. A equipe médica é variada (cirurgiões vasculares, ortopedistas, cirurgião geral, etc) não tendo horário pré-estabelecido de permanência nos setores de internamento e, portanto, a dificuldade em se manter a comunicação e o acesso para a discussão das intervenções clínicas. Como estratégia foi elaborado documento padrão da Farmácia Clínica, com diferenciação da cor do papel para facilitar a visualização pela equipe, mesmo assim não tivemos sucesso quanto à sinalização pelo prescritor.

Somado a essas barreiras, a dificuldade estrutural do hospital retarda as atividades relacionadas a reconciliação medicamentosa, pois, o hospital em estudo não dispõe de sistema informatizado que permita a prescrição eletrônica como porte da padronização do processo, para reverter isso, atualmente tem-se estudado e testado kits de ferramentas para auxiliar no desenvolvimento de programas robustos de reconciliação de medicamentos, pautados no Estudo de Melhoria da Qualidade de Reconciliação de Medicamentos em vários centros o MARQUIS (Stolldorf et al, 2020).

Existem evidências de que as intervenções Farmacêuticas são importantes e eficazes para reduzir a ocorrência de discrepâncias, pois o acompanhamento diário do Farmacêutico Clínico junto à equipe multidisciplinar, colabora no aumento da segurança e prevenção de eventos adversos (Miranda et al., 2012; Hellstrom et al, 2012; Leite et al, 2019).

\section{Conclusão}

Este estudo mostrou a prevalência de discrepâncias não intencionais da farmacoterapia do hospital estudado, os medicamentos envolvidos nas divergências e o perfil terapêutico dos pacientes internados na condição pós-cirúrgica, demonstrando a importância de processos como a reconciliação medicamentosas para a correção de erros que podem causar 
sérios danos aos pacientes. Ademais, este trabalho evidência que a investigação da história de uso de medicamentos é um processo complexo e que o farmacêutico deve ser um dos profissionais presentes para tal avaliação. Esses fatos sugerem a necessidade da implantação da reconciliação medicamentosa como uma prática organizacional e estratégica destinada a assegurar que os medicamentos usados pelo paciente em âmbito domiciliar, bem como os medicamentos do pós-operatório sejam prescritos correta e adequadamente.

\section{Referências}

Documento de referência para o Programa Nacional de Segurança do Paciente / Ministério da Saúde; Fundação Oswaldo Cruz; Agência Nacional de Vigilância Sanitária. Ministério da Saúde, 2014

Drenth-van Maanen, A. C., Spee, J., van Hensbergen, L., Jansen, P. A., Egberts, T. C., \& van Marum, R. J. (2011). A anamnese estruturada do uso de medicamentos revela dano iatrogênico devido a discrepâncias nos históricos de medicamentos em registros hospitalares e de farmácia. $J$ Am Geriatr Soc, 59 (10), 1976-1977.

Flamme-Obry, F., Belaiche, S., Hazzan, M., Ramdan, N., Noël, C., Odou, P., \& Décaudin, B. (2018). Impact du pharmacien clinicien sur la iatrogénie médicamenteuse chez le patient greffé rénal. Néphrologie \& Thérapeutique, 14(2), 91-98.

Greenwald, J. L., Halasyamani, L., Greene, J., LaCivita, C., Stucky, E., Benjamin, B., \& Williams, M. V. (2010). Making inpatient medication reconciliation patient centered, clinically relevant and implementable: a consensus statement on key principles and necessary first steps. Journal of Hospital Medicine, 5(8), $477-485$.

Hellström, L. M., Bondesson, Å., Höglund, P., \& Eriksson, T. (2012). Errors in medication history at hospital admission: prevalence and predicting factors. BMC clinical pharmacology, 12(1), 1-9.

Mc Comb, K. G., da Fonseca Meireles, S., \& Pinheiro, F. G. Qual é a importância do enfermeiro auditor para a melhoria da qualidade de assistência à saúde? A pluralidade do conhecimento na saúde, na educação e na tecnologia, 88.

Lombardi, N. F., Mendes, A. E. M., Lucchetta, R. C., Reis, W. C. T., Fávero, M. L. D., \& Correr, C. J. (2016). Análisis de las discrepancias encontradas durante la conciliación medicamentosa en la admisión de pacientes en unidades de cardiología: un estudio descriptivo. Revista Latino-Americana de Enfermagem, 24.

Markovic, M., Mathis, A. S., Ghin, H. L., Gardiner, M., \& Fahim, G. (2017). A comparison of medication histories obtained by a pharmacy technician versus nurses in the emergency department. Pharmacy and Therapeutics, 42(1), 41.

Mekonnen, A. B., McLachlan, A. J., \& Brien, J. A. (2016). Effectiveness of pharmacist-led medication reconciliation programmes on clinical outcomes at hospital transitions: a systematic review and meta-analysis. BMJ open, 6(2), e010003.

Mendes, A. E., Lombardi, N. F., Andrzejevski, V. S., Frandoloso, G., Correr, C. J., \& Carvalho, M. (2016). Medication reconciliation at patient admission: a randomized controlled trial. Pharmacy Practice (Granada), 14(1), 0-0.

Miranda, T. M. M., Petriccione, S., Ferracini, F. T., \& Borges Filho, W. M. (2012). Interventions performed by the clinical pharmacist in the emergency department. Einstein (São Paulo), 10, 74-78.

Nuez, C. R., Navarro, M. G., Valdivieso, J. G., Barrera, M. F., Sumalla, A. B., Rubí, J. S., \& Eroles, X. G. (2012). Efectividad de un programa de conciliación perioperatoria de la medicación crónica en pacientes de cirugía programada. Medicina Clínica, 139(15), 662-667.

Oliveira, S. T. de, Farias, P. de O., Drummond, B. M., Rodrigues, L. B., Reis, P. G. dos, Souza, L. de O., Oliveira, L. R. de, \& Miranda, V. F. de. (2018). Taxas de erro de prescrição e dispensação de um hospital público especializado em urgência e trauma. Rev Med Minas Gerais, 5, 61-68.

Pimenta, P. A., Santos, K. F. S., Silva, C. M. L., da Silva Passos, I., da Cunha Barros, I. M., \& de Carvalho Brito, G. (2020). Conciliação de medicamentos em um hospital de ensino de Sergipe: lições aprendidas na implementação de um serviço. Scientia Plena, 16(8).

Peixoto, A. M., Zimpel, S. A., Oliveira, A. C. A. D., Monteiro, R. L. S., \& Carneiro, T. K. G. (2017). Prevalência de amputações de membros superiores e inferiores no estado de Alagoas atendidos pelo SUS entre 2008 e 2015. Fisioterapia e Pesquisa, 24, 378-384.

Quélennec, B., Beretz, L., Paya, D., Blicklé, J. F., Gourieux, B., Andrès, E., \& Michel, B. (2013). Potential clinical impact of medication discrepancies at hospital admission. European journal of internal medicine, 24(6), 530-535.

Salanitro, A. H., Kripalani, S., Resnic, J., Mueller, S. K., Wetterneck, T. B., Haynes, K. T., \& Schnipper, J. L. (2013). Rationale and design of the multicenter medication reconciliation quality improvement study (MARQUIS). BMC health services research, 13(1), 1-12.

Santos, K. P. B. D., Luz, S. C. T. D., Mochizuki, L., \& d'Orsi, E. (2018). Carga da doença para as amputações de membros inferiores atribuíveis ao diabetes mellitus no Estado de Santa Catarina, Brasil, 2008-2013. Cadernos de Saúde Pública, 34.

Stolldorf, D. P., Mixon, A. S.., Auerbach, A. D., Aylor, A. R., Shabbir, H., Schnipper, J., \& Kripalani, S. (2020). Implementação e sustentabilidade de um kit de ferramentas de reconciliação de medicamentos: uma avaliação de métodos mistos. Jornal americano de farmácia do sistema de saúde: AJHP: jornal oficial da Sociedade Americana de Farmacêuticos do Sistema de Saúde, 77 (14), 1135-1143. 